\title{
Infusion of human umbilical cord-derived mesenchymal stem cells effectively relieves liver cirrhosis in DEN-induced rats
}

\author{
JINGXIN HONG $^{1 *}$, HUAJUN JIN ${ }^{2 *}$, JUNLING HAN $^{1,3^{*}}$, HUANZHANG HU ${ }^{2}$, JIAN LIU ${ }^{1}$, \\ LINFANG LI $^{2}$, YAO HUANG ${ }^{2}$, DANDAN WANG ${ }^{1}$, MENGCHAO WU $^{2}$, LUGUI QIU $^{3}$ and QIJUN QIAN ${ }^{2}$ \\ ${ }^{1}$ Union Stem Cell and Gene Engineering Co., Ltd., Tianjin Cord Blood Bank, \\ Tianjin 300384; ${ }^{2}$ Laboratory of Viral and Gene Therapy, Eastern Hepatobiliary Surgery Hospital, \\ Second Military Medical University, Shanghai 200438; ${ }^{3}$ Institute of Hematology, Blood Disease Hospital, \\ Peking Union Medical College and Chinese Academy of Medical Sciences, Tianjin 300020, P.R. China
}

Received June 22, 2013; Accepted January 6, 2014

DOI: $10.3892 / \mathrm{mmr} .2014 .1927$

\begin{abstract}
Cirrhosis is the long-term outcome of chronic hepatic injury and no effective therapy is currently available for this disease. Mesenchymal stromal cells (MSCs) are multipotent cells that are easily acquired and amplified, and may be potential candidates for cell therapy against cirrhosis. This study aimed to determine the therapeutic effects of human umbilical cord-derived MSCs (hUCMSCs) for the treatment of liver cirrhosis and identify an effective method for engrafting MSCs. The model of liver cirrhosis was established by induction of diethylnitrosamine (DEN) in rats. The isolated hUCMSCs were identified by morphology, flow cytometry and multilineage differentiation; they were injected into the vein of DEN-induced rats at varied cell doses and infusion times. Biochemical analyses of the serum and histopathological analysis of the liver tissues were performed to evaluate the therapeutic effects of hUCMSCs in all treatment groups. The results indicated that isolated hUCMSCs were capable of self-replication and differentiated into multiple lineages, including osteoblast-, adipocyte- and hepatocyte-like cells. Compared with the control group, administration of hUCMSCs at different cell doses and infusion times relieved DEN-induced cirrhosis to varying degrees. The therapeutic
\end{abstract}

Correspondence to: Dr Qijun Qian, Laboratory of Viral and Gene Therapy, Eastern Hepatobiliary Surgery Hospital, Second Military Medical University, Shanghai 200438, P.R. China

E-mail: qianqj@sino-gene.cn

Dr Lugui Qiu, Institute of Hematology and Blood Diseases Hospital, China Academy Medical Sciences and Peking Union Medical College, Tianjin 300020, P.R. China

E-mail: qiulg@ihcams.ac.cn

*Contributed equally

Key words: human umbilical cord-derived mesenchymal stem cells, liver cirrhosis, diethylnitrosamine effects of hUCMSCs on liver cirrhosis gradually improved with increased cell dose and infusion times. The improvement of cirrhosis was due to the capacity of hUCMSCs to breakdown collagen fibers in the liver. It was demonstrated that infusion of hUCMSCs effectively relieved liver cirrhosis by facilitating the breakdown of collagen fibers in a dose-dependent manner and multiple infusions caused a relatively greater improvement in cirrhosis compared with a single infusion of hUCMSCs.

\section{Introduction}

Cirrhosis is a consequence of chronic liver disease, it is characterized by scar tissue development, which replaces normal parenchyma and blocks the portal flow of blood through the liver subsequently hindering normal liver function (1). It can be divided into two categories, namely compensated and decompensated cirrhosis (2). The liver can compensate for a significant amount of damage through regeneration; however, the decompensation threshold is reached once the ability to replace damaged hepatocytes is lost. Ascites (fluid retention in the peritoneal cavity) is the most common complication resulting from decompensated cirrhosis and is associated with poor quality of life, increased risk of infection and a poor long-term outcome (3).

Cirrhosis is currently the 12th leading cause of mortality in the United States and is responsible for $>27,000$ deaths each year (4). Traditional treatments for cirrhosis commonly focus on preventing the progression and complications of the disease. In the advanced stages of cirrhosis, the only treatment option is liver transplantation, which is critically limited by the shortage of available liver donations required for the millions of patients with cirrhosis worldwide $(5,6)$. Thus, the requirement for a novel approach for treating liver cirrhosis is crucial.

It has been well documented that human mesenchymal stem cells, also known as mesenchymal stromal cells (MSCs) from different sources, have therapeutic potential for liver cirrhosis (7-9). Among them, bone marrow-derived MSCs (BMMSCs) can be injected into functional liver cells in vitro and differentiate into human hepatocytes without cell fusion in vivo (10). Previous studies have demonstrated that in rat and mouse models of carbon tetrachloride $\left(\mathrm{CCl}_{4}\right)$-induced 
Table I. Primers used for quantitative polymerase chain reaction in hepatogenic differentiation.

Primers

Sequence

Product

(bp)

\begin{tabular}{ll}
\hline$\alpha-F P$ & S: 5'-TACATTGACCACGTTCCAGC-3' \\
& A: 5'-ATGAGCACTGTTGCAGAGGA-3' \\
ALB & S: 5'-CTTGTTTTGCACAGCAGTCAG-3' \\
& A: 5'-CAAAAACATGTGTTGCTGATGA-3' \\
CK-18 & S: 5'-GTCAATCTGCAGAACGATGC-3' \\
& A: 5'-GAGCACTTGGAGAAGAAGGG-3' \\
CK-19 S: 5'-GTCGATCTGCAGGACAATCC-3' & 135 \\
& A: 5'-CCGCGACTACAGCCACTACT-3' \\
GAPDH & S: 5'-TCAGTGGTGGACCTGACCTG-3' \\
& A: 5'-TGCTGTAGCCAAATTCGTTG-3' \\
\hline
\end{tabular}

$\alpha$-FP, $\alpha$-fetoprotein; ALB, albumin; CK, cytokeratin.

liver fibrosis (a main pattern of the manifestation of cirrhosis), the application of BMMSCs showed potential therapeutic effects on the fibrotic process through their ability to differentiate into hepatocytes (11-15). In contrast to BMMSCs, human umbilical cord-derived MSCs (hUCMSCs) are more primitive and have lower immunogenicity and risk of viral transmission (16). Moreover, infusion of hUCMSCs in $\mathrm{CCl}_{4}$-induced cirrhotic rats alleviated liver fibrosis by inhibiting the expression of transforming growth factor- $\beta 1$, collagen type I and $\alpha$-smooth muscle actin (17). However, the optimal usage of hUCMSCs, particularly the cell dose and infusion times, have not been fully elucidated.

In this study, a model of liver cirrhosis in rats was established by the induction of diethylnitrosamine (DEN) and the therapeutic effects of hUCMSC infusion were investigated for reversing fibrosis using varied cell doses and infusion times. The treatment efficiency of hUCMSCs was examined through biochemical and histopathological analyses.

\section{Materials and methods}

\section{Isolation and culture of hUCMSCs}

Isolation. This study was approved by the ethics committee of the Tianjin Cord Blood Bank (Tianjin, China) and written informed consent was obtained from the subjects. Fresh human umbilical cord samples $(n=15)$ were collected at birth from full term deliveries and processed within $6 \mathrm{~h}$ following collection at $4^{\circ} \mathrm{C}$. MSCs were isolated according to the method described by Wang et al (18). Briefly, the umbilical cords were rinsed twice with Dulbecco's phosphate-buffered saline (D-PBS; no. 14190-144; Gibco-BRL, Carlsbad, CA, USA) treated with antibiotics (100 IU/ml penicillin and $100 \mu \mathrm{g} / \mathrm{ml}$ streptomycin) and minced into $1-2 \mathrm{~mm}^{3}$ fragments to expose Wharton's jelly. Fragments were then digested with $0.1 \%$ collagenase (20 $\mathrm{ml} 0.1 \%$ collagenase $\mathrm{II} / \mathrm{cm}$ umbilical cord) at $37^{\circ} \mathrm{C}$. The digested mixture was passed through a $100-\mu \mathrm{m}$ filter to obtain cell suspensions. Subsequent to removal of the supernatant fraction, the precipitate (dissociated MSCs) was washed with serum-free Dulbecco's modified Eagle's medium (DMEM)/F12 media (no. 12400-024; Gibco-BRL) twice and counted under an inverted microscope (ECLIPSE TE300; Nikon, Tokyo, Japan) using a hemocytometer (COUNTESSTM automated counter C10227, Invitrogen, Carlsbad, CA, USA). Cells were plated at a density of $1 \times 10^{4}$ cells $/ \mathrm{cm}^{2}$ in non-coated T-25 or T-75 cell culture flasks (Corning, San Jose, CA, USA).

Expansion. The suspended cells were removed after three days and adherent cells were additionally cultured. Cultured media was replaced every three days after initial plating. When the large fibroblast-like cell colonies developed after seven days, cultures were washed twice with PBS and the cells were detached with TrypLE ${ }^{\text {TM }}$ Select (no. 12563-029; Gibco-BRL) for $2-5 \mathrm{~min}$ at $37^{\circ} \mathrm{C}$. After centrifugation for $5 \mathrm{~min}$ at $300 \mathrm{x} \mathrm{g}$, cells were resuspended with DMEM/F12 supplemented with $10 \%$ fetal bovine serum (FBS) and incubated in $75-\mathrm{cm}^{2}$ culture flasks (Corning) for further expansion. The hUCMSCs between the 4th and 6th passages were collected for cell infusion.

\section{In vitro differentiation}

Osteogenic differentiation. To induce osteogenic differentiation, the hUCMSCs (4th passage, $1 \times 10^{4}$ cells $/ \mathrm{cm}^{2}$ ) were cultured in osteogenic medium (DMEM/F12 supplemented with $10 \%$ FBS, $0.1 \mathrm{mM}$ dexamethasone, $10 \mathrm{mM} \beta$-glycerol phosphate and $50 \mu \mathrm{M}$ ascorbic acid) for three weeks. Osteogenesis was then evaluated by bone sialoprotein (BSP) immunohistochemistry and Von Kossa staining.

Adipocyte differentiation. To induce adipogenesis differentiation, the hUCMSCs (4th passage, $1 \times 10^{4}$ cells $/ \mathrm{cm}^{2}$ ) were cultured in adipogenesis medium (DMEM/F12 supplemented with $10 \% \mathrm{FBS}, 1 \mu \mathrm{M}$ dexamethasone, $50 \mathrm{mg} / 1$ ascorbic acid and $100 \mathrm{mg} / \mathrm{l}$ isobutylmethyl xanthine) for three weeks. The obtained adipocyte-like cells were detected by oil red $\mathrm{O}$ staining, a method commonly utilized to identify the lipid content.

Hepatogenic differentiation. To induce hepatogenic differentiation, the hUCMSCs (4th passage, $1 \times 10^{4}$ cells $/ \mathrm{cm}^{2}$ ) were treated by a two-step protocol. Cells were cultured in the differentiation medium [DMEM/F12 supplemented with $10 \% \mathrm{FBS}, 20 \mathrm{ng} / \mathrm{ml}$ hepatocyte growth factor, 
$10 \mathrm{ng} / \mathrm{ml}$ basic fibroblast growth factor, $0.5 \mu \mathrm{M}$ dexamethasone, $0.61 \mathrm{mg} / \mathrm{ml}$ nicotinamide and $1 \mathrm{X}$ insulin/transferrin/sodium selenite mix (ITS)] for 14 days. Subsequently, the cells were transferred into a maturation medium [consisting of $20 \mathrm{ng} / \mathrm{ml}$ oncostatin $\mathrm{M}$ (OSM), $0.5 \mu \mathrm{M}$ dexamethasone and $1 \mathrm{X}$ ITS]. After four weeks, hepatogenesis was assessed by quantitative reverse transcription polymerase chain reaction (qRT-PCR) for the liver-associated genes listed in Table I. The relative expression of each gene was determined using GAPDH as an endogenous control and normalizing to that of L-02, a normal liver cell line.

Immunophenotype. The hUCMSCs (6th passage, $1 \times 10^{6}$ cells $/ \mathrm{cm}^{2}$ ) were incubated with fluorescein isothiocynate- or phycoerythrin (PE)-conjugated antibodies, including anti-CD29,-CD31, -CD90,-CD105, -HLA-DR (BD Biosciences, Sparks, MD, USA) and anti-CD73, -CD34, and -CD44 (AbD Serotec, Oxford, UK), for $30 \mathrm{~min}$ at $4^{\circ} \mathrm{C}$ in the dark. Allophycocyanin- or PE-labeled mouse immunoglobulin $\mathrm{G}$ was used as the negative control. After washing in PBS, cells were analyzed on a flow cytometer (FACSAria; BD Biosciences) collecting 10,000 events and the data were analyzed by cell analysis software (FACSCalibur; BD Biosciences).

Animal model of DEN-induced liver cirrhosis. All animal experiments were performed in adherence with the National Institutes of Health Guidelines on the Use of Laboratory Animals and approved by the Second Military Medical University Committee on Animal Care (Shanmghai, China). Male Sprague-Dawley rats, (age, 3.5 weeks; weight, 150-200 g) were purchased from the Shanghai Laboratory Animal Center (Shanghai, China). Rats were housed in an air-conditioned room at $25^{\circ} \mathrm{C}$ with specific pathogen-free conditions and were subjected to a $12 \mathrm{~h}$ light/dark cycle with access to chow and sterile water ad libitum. The rat model of liver cirrhosis was established by addition of $0.01 \%$ (v/v) DEN (Sigma-Aldrich, St. Louis, MO, USA) to the drinking water for four weeks.

hUCMSC administration. Rats were randomly divided into seven groups, including two control and five treatment groups. In the normal control group, rats were not induced with DEN and were injected with $1 \mathrm{ml}$ normal saline $(\mathrm{n}=10)$. In the DEN control group, rats were induced with DEN for four weeks and injected with $1 \mathrm{ml}$ normal saline $(\mathrm{n}=10)$. The treatment groups consisted of two series, namely the cell dose series and the infusion time series. The cell dose series included: The D1/T1 group in which rats received $1 \times 10^{6}$ hUCMSC infusion $(n=15)$; the D3 group in which rats received $3 \times 10^{6}$ hUCMSC infusion $(n=15)$; and the D6 group in which rats received $6 \times 10^{6}$ hUCMSC infusion $(n=15)$. The infusion time series included: The D1/T1 group (abovementioned); the T3 group in which rats received hUCMSC infusion three times at a dose of $1 \times 10^{6}$ cells $/ \mathrm{cm}^{2}$ once every three days $(\mathrm{n}=15)$; and the T6 group in which rats received hUCMSCs infusion six times at a dose of $1 \times 10^{6}$ cells $/ \mathrm{cm}^{2}$ once every three days $(n=15)$. Prior to infusion, the hUCMSCs were collected and suspended in $1 \mathrm{ml}$ normal saline and then the cell suspensions were injected intravenously via the tail vein. Rats were sacrificed via $2 \%$ pentobarbital anaesthesia injected through the abdominal cavity injection after four weeks and the liver tissues and blood samples from the heart were collected.

Biochemical examination. The biochemical parameters in the serum, including total bilirubin (TBIL), total protein (TP), albumin (ALB), globulin (GLB), alanine aminotransferase (ALT), aspartate aminotransferase (AST), glutamyl peptidase (GGT) and alkaline phosphatase (ALP) were analyzed with an automated biochemistry analyzer (XL-300; Erba Mannheim, Mannheim, Germany) at the clinical laboratory of the Eastern Hepatobiliary Surgery Hospital (Shanghai, China).

Histopathological analysis. The collected liver samples were fixed in $10 \%$ formaldehyde for $24 \mathrm{~h}$ at room temperature. Serial $5-\mu \mathrm{m}$ sections were stained with hematoxylin and eosin for routine histopathological analysis. The paraffin sections were stained with the collagen fibers kit (Genmed Scientifics, Inc., Arlington, MA, USA) for the quantitative analysis of collagen. The degree of hepatic fibrosis was assessed by two pathologists according to the METAVIR scoring system: 0, no fibrosis; I, perivenular and/or pericellular fibrosis; II, septal fibrosis; III, incomplete cirrhosis; and IV, complete cirrhosis (19).

Collagen detection. Relative expression levels of collagen type I $\alpha 1$ chain gene (Colla1) in rats from all groups was quantitatively analyzed by qPCR. The total RNA of three randomly selected liver samples either from the two control groups or the hUCMSC treatment groups were extracted using TRIzol reagent and the three independently extracted RNAs from the same group were equally mixed to form a bulk. Subsequently, the first strand of cDNA of each bulk was synthesized using a primescript 1st strand cDNA Synthesis kit and qPCR was performed using SYBR Premix Ex Taq (Takara Biotechnology, Inc., Dalian, China) with a PCR amplification cycle of $94^{\circ} \mathrm{C}$ for $60 \mathrm{sec}, 94^{\circ} \mathrm{C}$ for $5 \mathrm{sec}$ and $60^{\circ} \mathrm{C}$ for $30 \mathrm{sec}$, for 40 cycles. The relative Colla1 expression of all test groups was determined using GAPDH as an endogenous control and normalized to that of the normal group (Table I). Each sample was assayed in duplicate $(n=3)$.

Statistical analysis. All data are expressed as the mean \pm standard deviation. An independent Student's t-test was used to analyze the variation of two selected groups. $\mathrm{P}<0.05$ was considered to indicate a statistically significant difference.

\section{Results}

hUCMSCs are capable of self-replication and differentiating into multiple lineages. MSCs isolated from the human umbilical cord rapidly formed colonies, which homogeneously exhibited fibroblast-like morphology at a lower confluence on day one, while growing to confluence on day seven after initial plating (Fig. 1A). Flow cytometric analyses indicated that the MSC-specific markers, including adhesion molecules (CD29/CD44), mesenchymal cell [CD73 (SH3)/CD105 (SH2)] and stem cell markers (CD90) (Fig. 1B), were expressed, but the surface antigens of hematopoietic cells, such as CD31, CD34 and HLA-DR (Fig. 1B), were not detected. These fibroblast-like 
Table II. Values of biochemical indictors in the serum of the different groups.

\begin{tabular}{lcccccccc}
\hline Groups & TBIL $(\mu \mathrm{mol} / \mathrm{l})$ & TP $(\mathrm{g} / \mathrm{l})$ & ALB $(\mathrm{g} / \mathrm{l})$ & GLB $(\mathrm{g} / \mathrm{l})$ & ALT $(\mathrm{IU} / \mathrm{l})$ & AST $(\mathrm{IU} / \mathrm{l})$ & GGT $(\mathrm{IU} / \mathrm{l})$ & ALP $(\mathrm{IU} / \mathrm{l})$ \\
\hline Normal & $0.83 \pm 0.13^{\mathrm{b}}$ & $60.95 \pm 2.48$ & $40.33 \pm 1.24$ & $20.75 \pm 1.26$ & $30.65 \pm 6.47^{\mathrm{b}}$ & $76.10 \pm 8.53^{\mathrm{a}}$ & $0.25 \pm 0.50^{\mathrm{b}}$ & $114.00 \pm 9.20^{\mathrm{a}}$ \\
DEN & $5.67 \pm 1.63$ & $57.33 \pm 1.33$ & $37.80 \pm 1.56$ & $19.33 \pm 0.58$ & $132.30 \pm 30.26$ & $241.73 \pm 40.29$ & $48.33 \pm 19.66$ & $393.00 \pm 76.24$ \\
D1/T1 & $1.09 \pm 0.31^{\mathrm{b}}$ & $57.59 \pm 2.86$ & $39.20 \pm 2.00$ & $18.38 \pm 1.96$ & $52.93 \pm 16.38^{\mathrm{b}}$ & $145.78 \pm 25.24^{\mathrm{a}}$ & $4.31 \pm 4.69^{\mathrm{b}}$ & $210.94 \pm 56.85^{\mathrm{a}}$ \\
D3 & $1.16 \pm 0.36^{\mathrm{b}}$ & $58.50 \pm 1.79$ & $40.97 \pm 1.88$ & $17.5 \pm 1.72$ & $57.01 \pm 23.50^{\mathrm{b}}$ & $132.24 \pm 36.65^{\mathrm{a}}$ & $4.00 \pm 4.37^{\mathrm{b}}$ & $204.69 \pm 48.84^{\mathrm{a}}$ \\
D6 & $0.90 \pm 0.38^{\mathrm{b}}$ & $59.66 \pm 2.13$ & $40.58 \pm 2.82$ & $19.09 \pm 2.51$ & $55.36 \pm 19.84^{\mathrm{b}}$ & $132.11 \pm 39.15^{\mathrm{a}}$ & $2.27 \pm 2.61^{\mathrm{b}}$ & $182.45 \pm 32.48^{\mathrm{a}}$ \\
T3 & $1.07 \pm 0.25^{\mathrm{b}}$ & $63.57 \pm 1.65$ & $41.50 \pm 1.23$ & $22.33 \pm 1.15$ & $46.57 \pm 8.41^{\mathrm{b}}$ & $159.87 \pm 39.90^{\mathrm{a}}$ & $3.17 \pm 4.36^{\mathrm{b}}$ & $128.33 \pm 6.00^{\mathrm{a}}$ \\
T6 & $1.53 \pm 0.12^{\mathrm{b}}$ & $64.50 \pm 4.34$ & $43.03 \pm 1.86$ & $21.33 \pm 2.31$ & $49.13 \pm 7.20^{\mathrm{b}}$ & $151.60 \pm 56.74^{\mathrm{a}}$ & $1.67 \pm 1.15^{\mathrm{b}}$ & $110.00 \pm 39.25^{\mathrm{a}}$ \\
\end{tabular}

${ }^{\mathrm{a}} \mathrm{P}<0.05$ and ${ }^{\mathrm{b}} \mathrm{P}<0.01$. Data are expressed as the mean \pm standard deviation. Values of all groups were compared with those of the DEN control group. DEN, diethylnitrosamine; TBIL, total bilirubin; TP, total protein; ALB, albumin; GLB, globulin; ALT, alanine aminotransferase; AST, aspartate aminotransferase; GGT, glutamyl peptidase; ALP, alkaline phosphatase; D1/T1, 1x10 $0^{6}$ hUCMSC infusion; D3, 3x 10 ${ }^{6}$ hUCMSC infusion; D6, 5x $10^{6}$ hUCMSC infusion; T3, $1 \times 10^{6}$ hUCMSC infusion three times once every three days; T6, $1 \times 10^{6}$ hUCMSC infusion six times once every three days.

$\mathbf{A}$

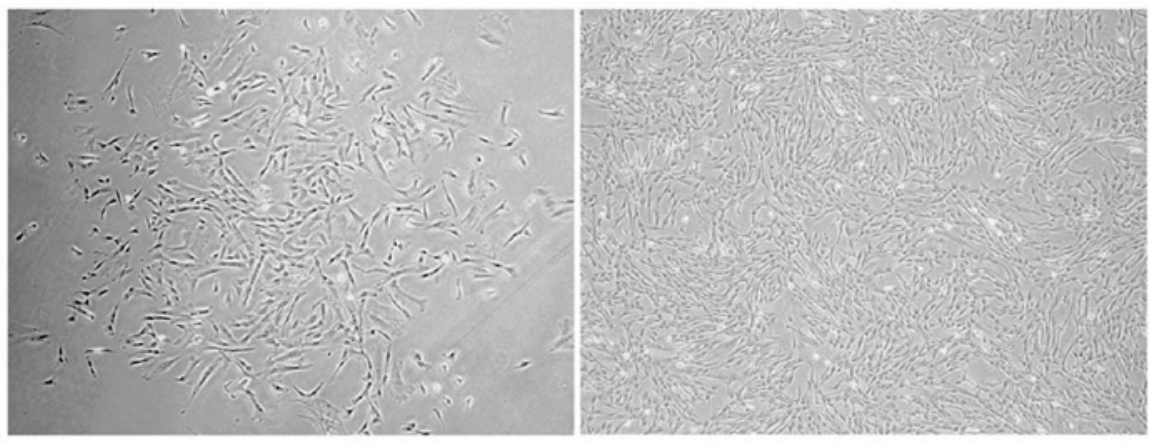

B

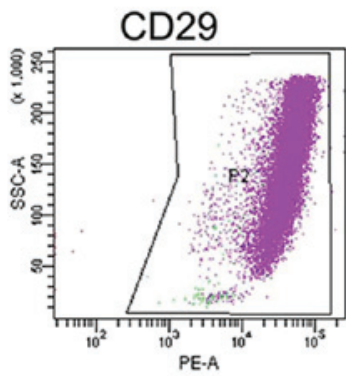

CD105

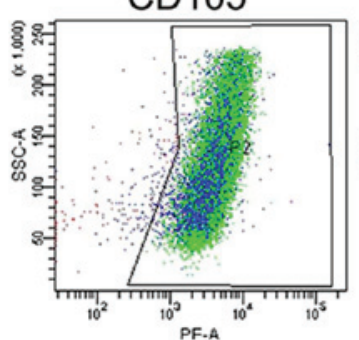

CD44

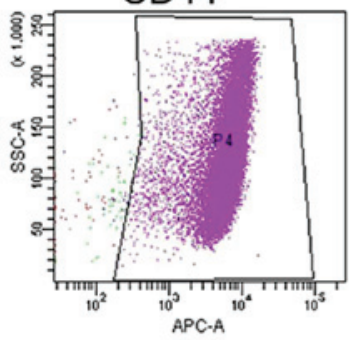

CD31

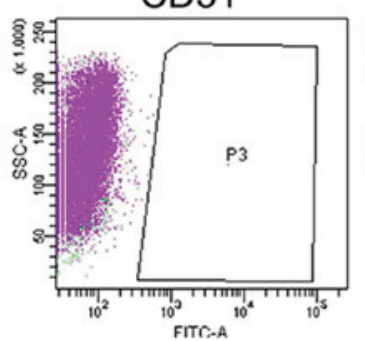

CD73

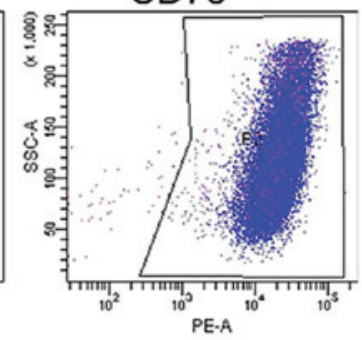

CD34

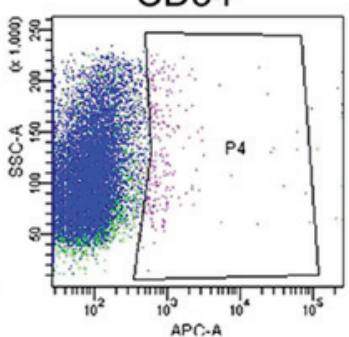

CD90

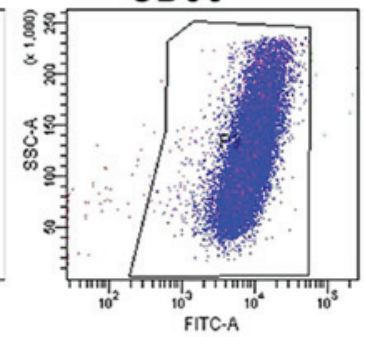

HLA-DR

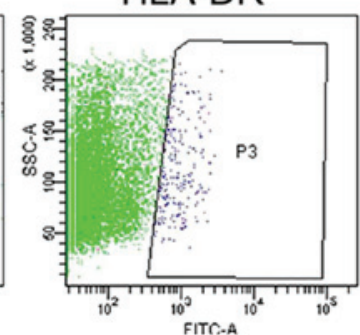

Figure 1. Flow cytometric analyses of isolated hUCMSCs. (A) Morphology of isolated hUCMSCs on day one (left) and day seven (right) (magnification, x200). (B) Multimarker analyses using flow cytometry. hUCMSCs, human umbilical cord-derived mesenchymal stromal cells..

MSCs were capable of self-replication through several passages and their duplication time ranged from 32 to $45 \mathrm{~h}$.

Under the induction of osteogenic medium for three weeks, the hUCMSCs differentiated into osteoblast-like cells that were positively expressed in BSP immunohistochemistry and Von Kossa staining (Fig. 2A). Under the induction of adipogenesis medium for three weeks, the hUCMSCs differentiated into adipocyte-like cells that were positively stained in oil red O (Fig. 2B). Under the induction of hepatic medium for four weeks, the hUCMSCs lost the primary fibroblastic morphology. hUCMSCs developed a cube-like morphology at the 1st stage and exhibited hepatocyte morphology and 
Table III. Cirrhotic grade determined by pathological analysis.

\begin{tabular}{lccccc}
\hline Group & Grade 0 & Grade I & Grade II & Grade III & Grade IV \\
\hline Normal & 10 & 0 & 0 & 0 & 2 \\
DEN $^{\text {a }}$ & 0 & 0 & 0 & 1 & 0 \\
D1/T1 & 3 & 8 & 3 & 0 & 0 \\
D3 & 4 & 6 & 5 & 0 & 0 \\
D6 & 8 & 5 & 2 & 0 & 0 \\
T3 & 9 & 6 & 0 & 0 & 0 \\
T6 & 11 & 4 & 0 & 0 \\
\hline
\end{tabular}

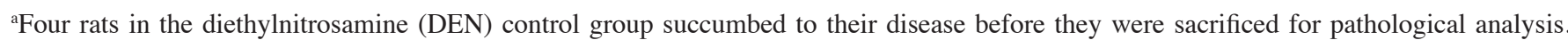
D1/T1, $1 \times 10^{6}$ hUCMSC infusion; D3, 3x $10^{6}$ hUCMSC infusion; D6, 5x $10^{6}$ hUCMSC infusion; T3, $1 \times 10^{6}$ hUCMSC infusion three times once every three days; T6, $1 \times 10^{6}$ hUCMSC infusion six times once every three days.

A

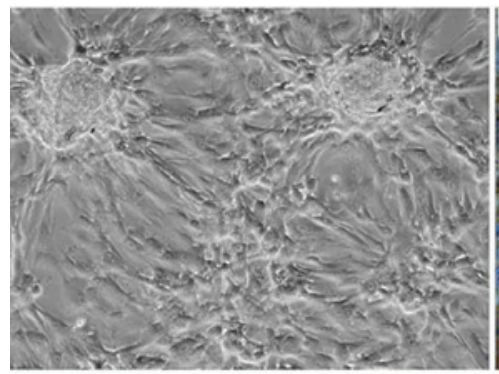

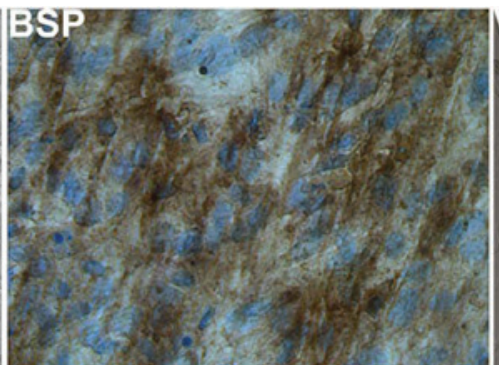

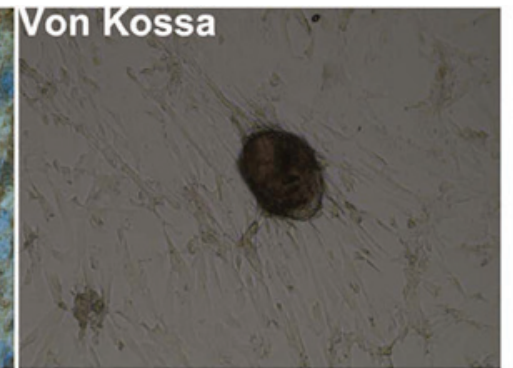

B
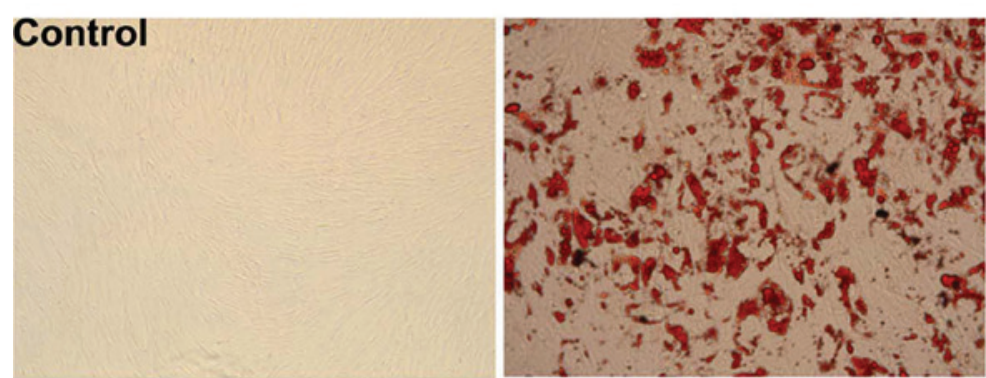

C
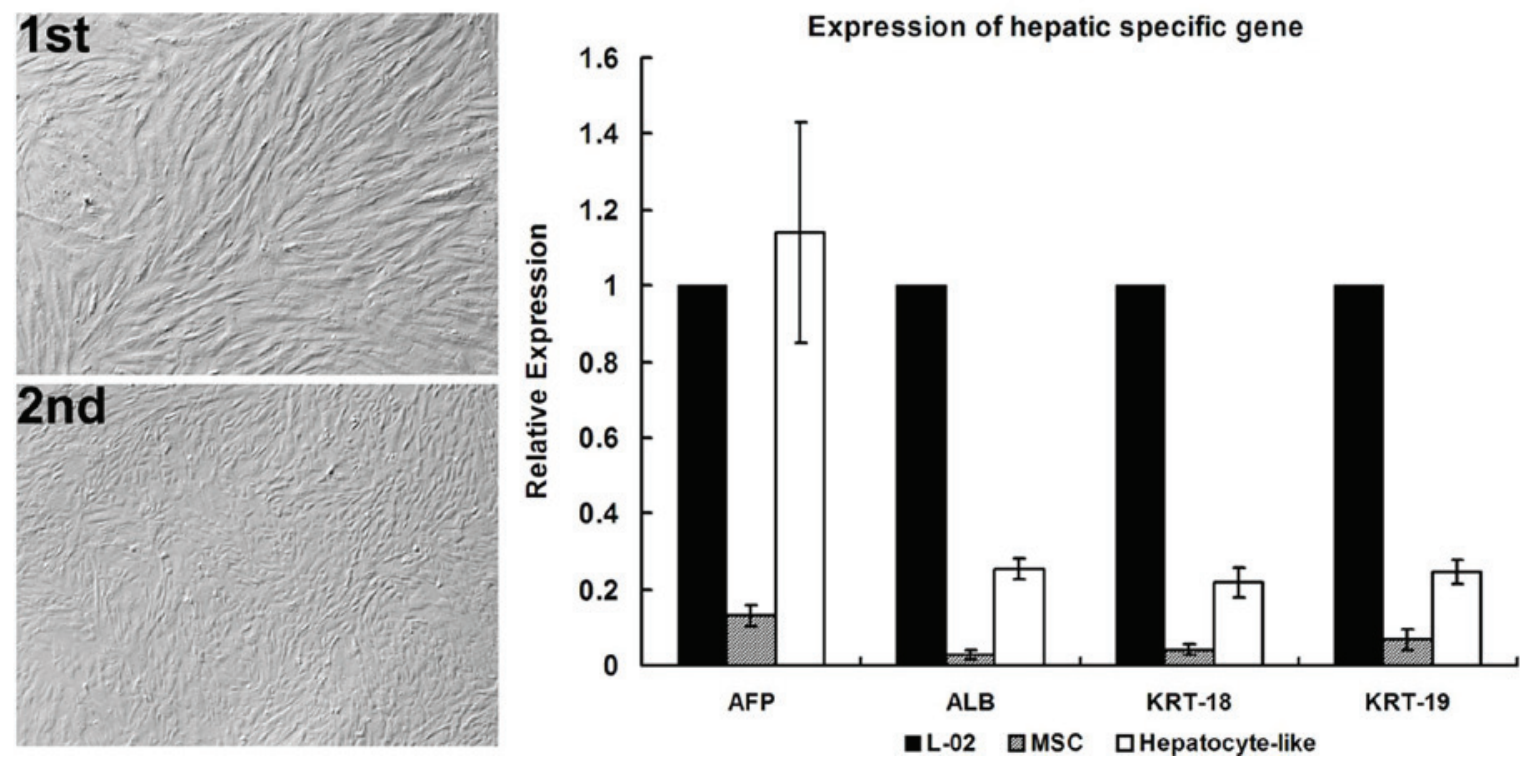

Figure 2. Multilineage differentiation of isolated hUCMSCs. (A) Osteoblast differentiation was identified by BSP immunohistochemistry and Von Kossa staining (magnification, x200). (B) Adipocyte differentiation was detected by oil red O staining (magnification, x200). (C) Two-stage hepatocyte differentiation (magnification,x200).hUCMSCs, humanumbilicalcord-derivedmesenchymalstromalcells;BSP,bonesialoprotein;AFP, $\alpha$-fetoprotein;ALB,albumin;KRT,keratin. 


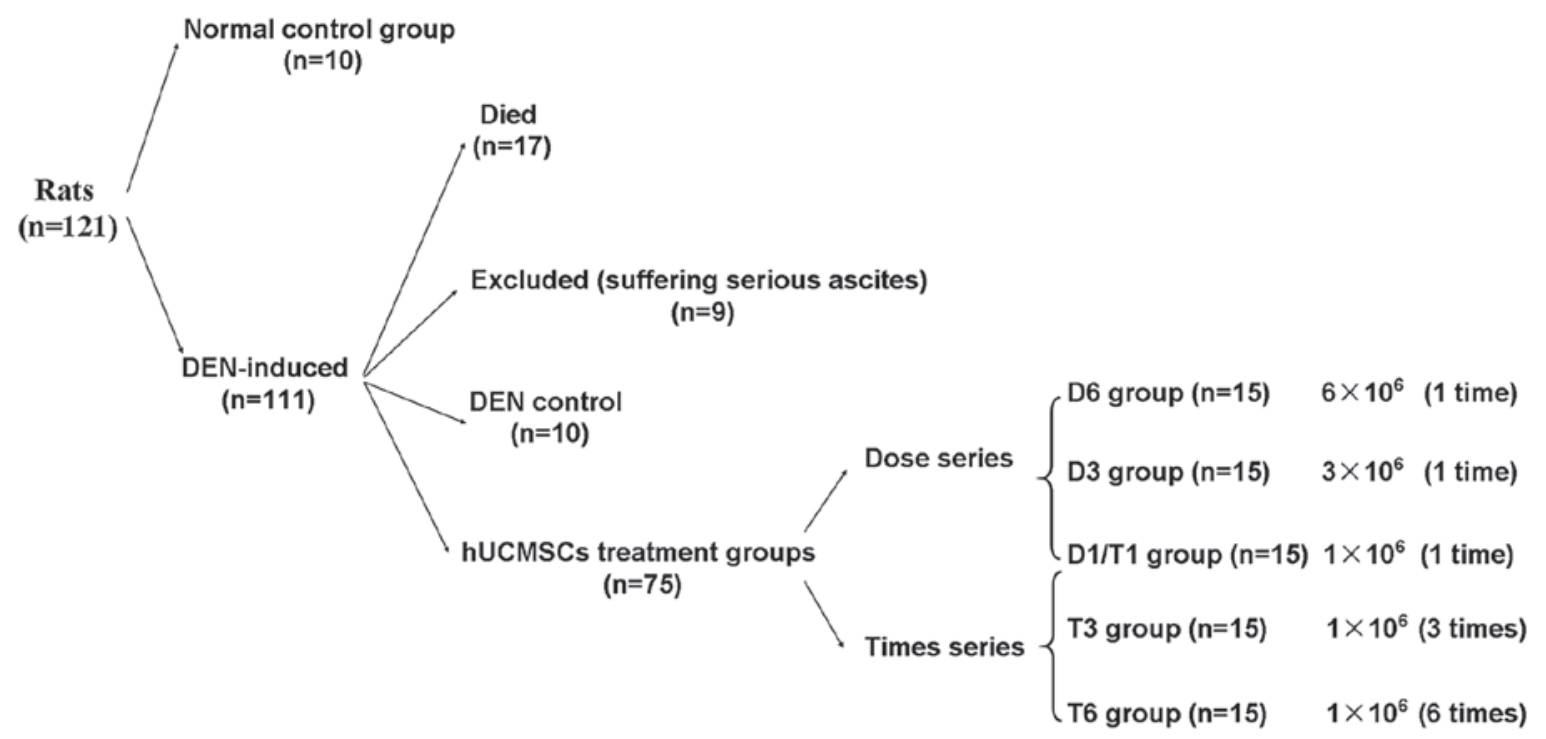

Figure 3. Flow chart summarizing the course of the animal experiment and the different groups. DEN, diethylnitrosamine; D1/T1, 1x106 hUCMSC infusion;

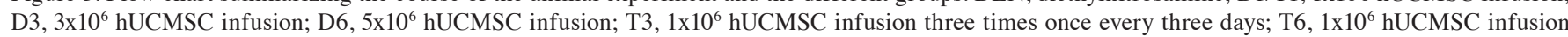
six times once every three days.

A
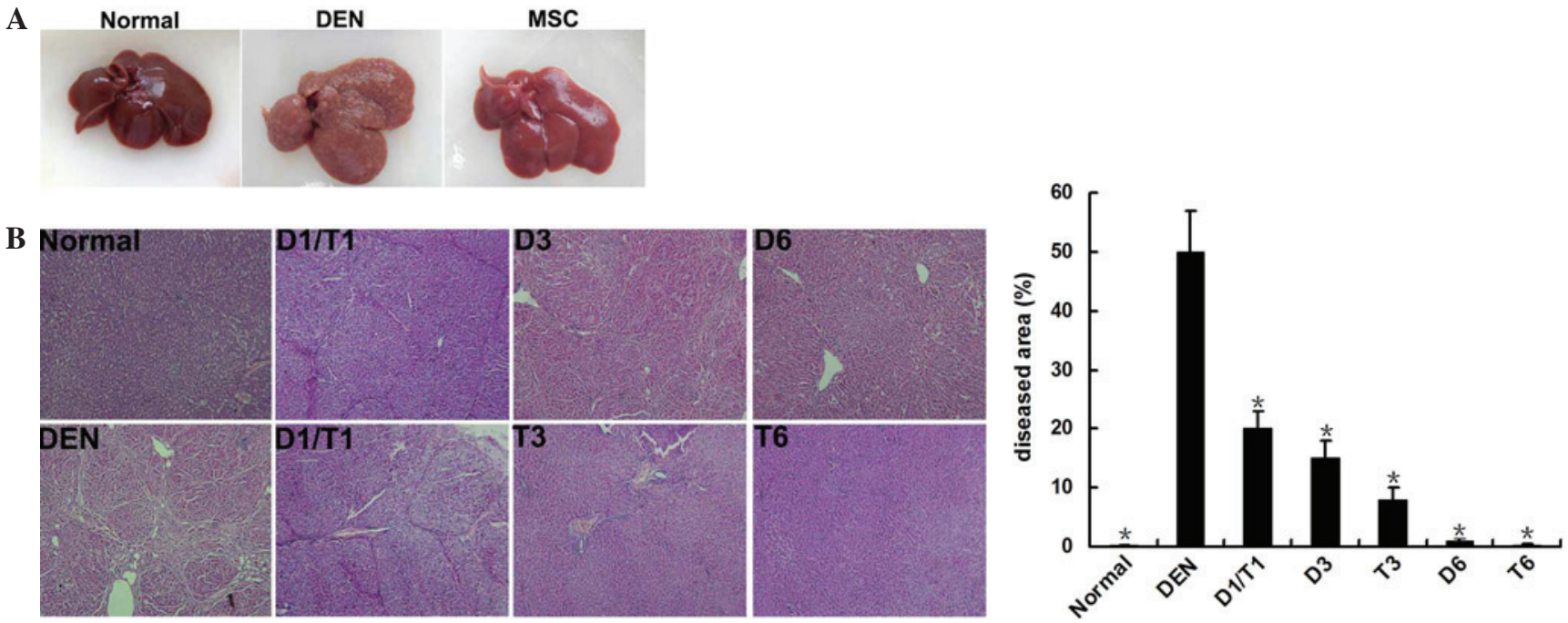

Figure 4. Pathological analyses of liver tissues. (A) Liver morphology. Normal, rat in the normal control group; MSC, a representative rat chosen from the T6 group. (B) Pathological analyses of liver tissues from various groups (magnification, $\mathrm{x} 200$ ). The quantitative result is shown on the right. ${ }^{*} \mathrm{P}<0.05$ compared with the DEN control group. DEN, diethylnitrosamine, MSC, mesenchymal stromal cells. Normal, rat in the normal control group; MSC, a representative rat chosen from the T6 group.

expressed hepatic-specific genes at the 2nd stage (Fig. 2C). These results indicated that the isolated hUCMSCs were able to differentiate into multiple lineages.

DEN successfully induces liver fibrosis in rats. At the end of induction, $14.2 \%$ (17 of the 120 rats) of DEN-induced rats succumbed to their disease and $6.7 \%$ ( 8 of the 120 rats) of the rats that survived exhibited serious ascites (Fig. 3). The body weight of the DEN-induced rats was markedly lower than that of the normal rats (data not shown). The livers of DEN-induced rats appeared coarser and almost bloodless with numerous small regenerative nodules (Fig. 4). By contrast, the livers of the normal group were smooth, lustrous and reddish on the surface. Furthermore, repeated DEN intake led to a marked increase of TBIL, ALT, AST, GGT and
ALP levels in the blood, suggesting that the liver function of DEN-induced rats was damaged (Table II). Histopathological analysis of the liver tissues identified a thick fibrous septa extending from the portal tracts into the liver parenchyma of DEN-induced rats (Fig. 4). These results indicated that liver fibrosis was successfully induced when rats were treated with DEN via the drinking water, which was consistent with a previous finding that liver fibrosis was induced by weekly intraperitoneal injections of DEN (20). Subsequently, the remaining $85 \mathrm{DEN}$-induced rats (except the 8 rats exhibiting serious ascites) were randomly divided into the DEN control group and 5 hUCMSCs treatment groups (Fig. 3).

hUCMSCs infusion ameliorates biochemical indictors. Four weeks following infusion, the biochemical indictors in the rat 

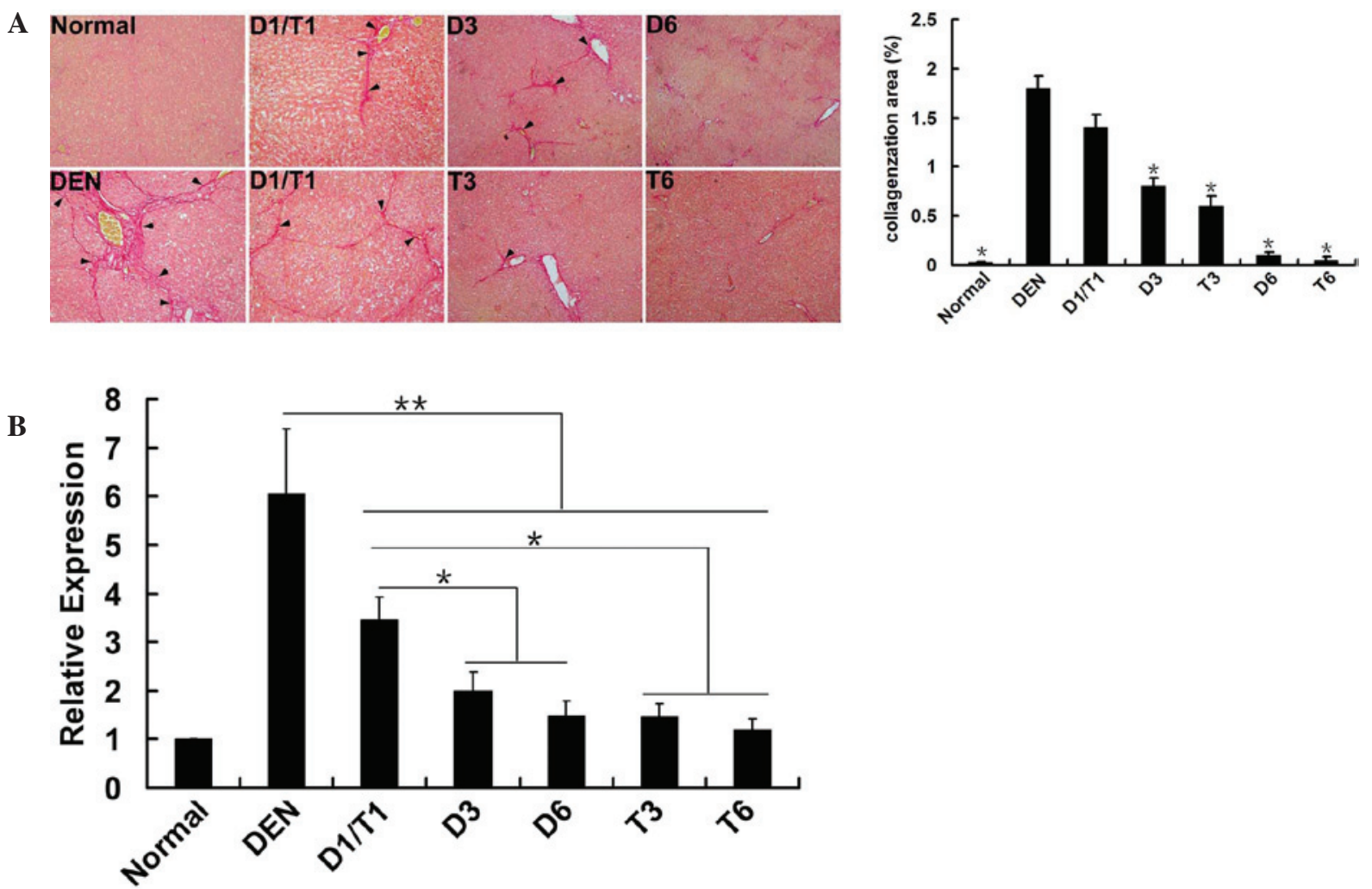

Figure 5. Analysis of collagen fiber. (A) Pathological analysis of liver tissues from various groups using collagen fiber staining. The arrows indicate the collagen deposition (magnification, $\mathrm{x} 200$ ). The quantitative result is shown on the right. ${ }^{*} \mathrm{P}<0.05$ compared with the DEN control group. (B) The relative collagen type I $\alpha 1$ chain gene expression was measured by quantitative polymerase chain reaction using GAPDH as the endogenous control and the detected expression values were normalized to that of normal liver tissue. ${ }^{*} \mathrm{P}<0.05$ and ${ }^{* *} \mathrm{P}<0.01$. DEN, diethylnitrosamine; D1/T1, $1 \times 106 \mathrm{hUCMSC}$ infusion; $\mathrm{D} 3,3 \mathrm{x} 10^{6} \mathrm{hUCMSC}$ infusion; D6, $5 \times 10^{6}$ hUCMSC infusion; T3, $1 \times 10^{6}$ hUCMSC infusion three times once every three days; T6, 1x10 ${ }^{6}$ hUCMSC infusion six times once every three days.

serum were measured to identify the capacity of hUCMSC infusion on improving liver function. As shown in Table II, serum markers, including TBIL, ALT, AST, GGT and ALP, significantly decreased in all hUCMSCs treatment groups compared with those of the DEN control group $(\mathrm{P}<0.01)$. Compared with the normal control group, which was not induced by DEN, the values of TBIL decreased quicker than the remaining serum markers, the levels of TBIL almost returned to normal even in the low dose group (D1/T1 group). The values of ALT and AST also decreased rapidly. However, they remained marginally higher than the normal level even in the high dose treatment groups (D6 and T6 groups). The values of GGT and ALP decreased relatively slower and gradually returned to a normal level with prolonged cell dose and infusion times. Comparison of the paired groups that received the same dose of hUCMSCs, but at different infusion times (i.e., D3 and T3, D6 and T6) indicated the GGT and ALP levels in the $\mathrm{T} 3$ and $\mathrm{T} 6$ groups were relatively higher than those in the D3 and D6 groups.

hUCMSC infusion reverses liver fibrosis. Histopathological analysis was performed to directly investigate the capacity of hUCMSC infusion for reversing liver fibrosis. The histopathology of liver tissues between the hUCMSC treatment and the control groups was compared. The results demonstrated that the livers of rats that survived treatment in the DEN control group $(n=6$, four rats in the group succumbed within two weeks) were seriously fibrotic, displaying disruption of tissue architecture, extension of fibers and large fibrous septa formation (Fig. 4). In the DEN control group two rats exhibited grade III liver fibrosis and four exhibited grade IV fibrosis (Table III). By contrast, liver fibrosis was greatly repaired following one infusion of hUCMSCs at a dose of $1 \times 10^{6}$ cells $/ \mathrm{cm}^{2}$ (D1/T1 groups), exhibiting relatively normal architecture of the liver parenchyma (Fig. 4). With prolonged cell dose or infusion times, liver fibrosis decreased or disappeared (Fig. 4) and the grade of liver fibrosis was close to normal (grade 0). Consistent with the results obtained from the biochemical analysis, the fibrotic state of rats in the T3 and T6 groups was less severe than that of the D3 and D6 groups (Table III and Fig. 4).

hUCMSC infusion facilitates the breakdown of collagen fibers. Collagen fiber staining was performed on the rat liver tissues. The results showed that the livers from the DEN control group displayed marked collagen distribution along the edge of the liver lobules (Fig. 5A). In the hUCMSC treatment groups, collagen deposition was markedly decreased (Fig. 5A), particularly in the D6 and T6 groups, which displayed almost no collagen accumulation compared with the DEN control group (Fig. 5A).

Furthermore, the expression of Colla1 in three randomly selected liver samples from each group were quantified by qPCR. The results showed that the Colla1 expression of rats in the hUCMSC treatment groups was significantly decreased compared with that in the DEN control group $(\mathrm{P}<0.05)$. With 
increased cell dose or infusion time, the expression levels of Collal were gradually decreased to that of the normal group (Fig. 5B).

\section{Discussion}

MSCs are capable of self-renewal while maintaining their potential to differentiate into multiple lineages (21). At present, MSCs have been isolated from bone marrow, umbilical cord, adipose tissue, adult muscle and the dental pulp of deciduous baby teeth (22). Among these, hUCMSCs are the youngest cells with more primitive properties than that of cells from other origins; therefore, hUCMSCs may be a useful source of MSCs for clinical application (16). In this study, the characteristics of hUCMSCs and their potential to differentiate into osteoblast-, adipocyte- and hepatocyte-like cells were identified. In addition, their ability to treat DEN-induced liver fibrosis in rats was investigated. It was demonstrated that infusion of hUCMSCs effectively relieved DEN-induced liver fibrosis in a dose-dependent manner by facilitating the breakdown of collagen fibers.

Biochemical indicators in the serum are clinically important for determining liver function (23). To a certain extent, the values of ALB, TP and GLB can reflect the capacity of the liver to synthesize protein, while the values of TBIL, ALT, AST, GGT and ALP can reflect damage to the liver. In this study, the values of ALB, TP and GLB were similar between the normal control group and the DEN control group, whereas the values of TBIL, ALT, AST, GGT and ALP were distinctly different between the two groups. Therefore, DEN-induced rats demonstrated compensated cirrhosis. Histopathology showed that the liver was heavily scarred, but could still perform several crucial functions. As the values of TBIL, ALT and AST were recovered to different degrees by the infusion of hUCMSCs, it was hypothesized that hUCMSC-based treatment may be effective for compensated cirrhosis.

Although the potential of MSCs to relieve liver cirrhosis has been repeatedly verified $(10-15,17)$, the effective infusion dosage and times have not been fully elucidated. In this study, it was demonstrated that the therapeutic effects of hUCMSCs in the T3 and T6 groups were relatively greater than that in the D3 and D6 groups, indicating that multiple hUCMSC infusions are more effective than a single infusion. The possible underlying mechanism is that multiple infusions of MSCs maintain the greatest number of healthy cells, which mediates the reduction in fibrosis. Notably, liver fibrosis was gradually relieved with increased infusion time. The results indicated that the infusion of hUCMSCs to immune-competent rats does not induce a strong immune response, which is consistent with the view that hUCMSCs possess low immunogenicity and are safe as regenerative medicinal treatments (16).

Currently, the underlying mechanisms of MSCs for treating liver cirrhosis have not been fully elucidated $(24,25)$. Previous studies have suggested that the ability of MSCs to differentiate into hepatocytes occurs during the liver recovery process, as engrafted human MSCs can express human $\alpha$-fetoprotein, ALB and cytokeratin-18 $(10,14,26)$. By contrast, opposing results have been obtained which reject this theory (17). Additionally, other mechanisms, including paracrine effects have been studied $(24,25)$. It has been demonstrated that MSCs can secrete a variety of bioactive cytokines or growth factors, including interleukin-10, tumor necrosis factor- $\alpha$, hepatocyte growth factor and nerve growth factor, to induce apoptosis of hepatic stellate cells (the major source of fibrillar collagens) and consequently facilitate the regeneration of the liver (26-29). Thus, paracrine effects may exhibit a major role since the transplanted hUCMSCs have been found to repair liver fibrosis by facilitating the breakdown of collagen fibers. Considering the different characteristics of cirrhosis, it is possible that infused MSCs may relieve liver cirrhosis through different mechanisms. They function mainly through paracrine effects for treating compensated cirrhosis or via hepatocyte-differentiation for decompensated cirrhosis.

In conclusion, this study demonstrated that hUCMSCs can differentiate into multiple lineages. Infusion of hUCMSCs can effectively relieve liver cirrhosis in a dose-dependent manner and multiple infusions caused a relatively greater reversal of cirrhosis compared with a single infusion of hUCMSCs. These results may provide an insight into the potential clinical application of hUCMSCs for treating liver cirrhosis.

\section{Acknowledgements}

This study was supported by the National Natural Science Funds for Distinguished Young Scholar (grant no. 30925037); the National Natural Science Foundation of China (grant no. 81001013); the National S\&T Major Special Project on New Drug Innovation of China (grant no. 2011ZX09102-010-02); and the Major State Basic Research Development Program (973 Programs, grant no. 2010CB529900-G).

\section{References}

1. Friedman SL: Liver fibrosis - from bench to bedside. J Hepatol 38 (Suppl 1): S38-S53, 2003.

2. Gines P, Quintero E, Arroyo V, Teres J, et al: Compensated cirrhosis: natural history and prognostic factors. Hepatology 7: 122-128, 1987.

3. Moore KP and Aithal GP: Guidelines on the management of ascites in cirrhosis. Gut 55 (Suppl 6): vi1-vi12, 2006.

4. Sanyal AJ, Mullen KD and Bass NM: The treatment of hepatic encephalopathy in the cirrhotic patient. Gastroenterol Hepatol (NY) 6 (Suppl 8): S1-S12, 2010.

5. Murray KF, Carithers RL Jr and AASLD: AASLD practice guidelines: Evaluation of the patient for liver transplantation. Hepatology 41: 1407-1432, 2005.

6. Muraca M: Evolving concepts in cell therapy of liver disease and current clinical perspectives. Dig Liver Dis 43: 180-187, 2011.

7. Lee KD, Kuo TK, Whang-Peng J, et al: In vitro hepatic differentiation of human mesenchymal stem cells. Hepatology 40: 1275-1284, 2004.

8. Chen Y, Dong XJ and Zhang GR: In vitro differentiation of mouse bone marrow stromal stem cells into hepatocytes induced by conditioned culture medium of hepatocytes. J Cell Biochem 102: 52-63, 2007.

9. Mohsin S, Shams S, Ali Nasir G, et al: Enhanced hepatic differentiation of mesenchymal stem cells after pretreatment with injured liver tissue. Differentiation 81: 42-48, 2011.

10. Sato Y, Araki H, Kato J, et al: Human mesenchymal stem cells xenografted directly to rat liver are differentiated into human hepatocytes without fusion. Blood 106: 756-763, 2005.

11. Fang B, Shi M, Liao L, et al: Systemic infusions of FLK1(+) mesenchymal stem cells ameliorate carbon tetrachloride-induced liver fibrosis in mice. Transplantation 78: 83-88, 2004.

12. Zhao DC, Lei JX, Chen R, et al: Bone marrow-derived mesenchymal stem cells protect against experimental liver fibrosis in rats. World J Gastroenterol 11: 3431-3440, 2005. 
13. Abdel Aziz MT, Atta HM, Mahfouz S, et al: Therapeutic potential of bone marrow-derived mesenchymal stem cells on experimental liver fibrosis. Clin Biochem 40: 893-899, 2007.

14. Chang YJ, Liu JW, Lin PC, et al: Mesenchymal stem cells facilitate recovery from chemically induced liver damage and decrease liver fibrosis. Life Sci 85: 517-525, 2009.

15. Lee MJ, Jung J, Na KH, et al: Anti-fibrotic effect of chorionic plate-derived mesenchymal stem cells isolated from human placenta in a rat model of $\mathrm{CCl}_{4}$-injured liver: potential application to the treatment of hepatic diseases. J Cell Biochem 111: 1453-1463, 2010.

16. Fan CG, Zhang QJ and Zhou JR: Therapeutic potentials of mesenchymal stem cells derived from human umbilical cord. Stem Cell Rev 7: 195-207, 2011.

17. Tsai PC, Fu TW, Chen YM, et al: The therapeutic potential of human umbilical mesenchymal stem cells from Wharton's jelly in the treatment of rat liver fibrosis. Liver Transpl 15: 484-495, 2009.

18. Wang HS, Hung SC, Peng ST, et al: Mesenchymal stem cells in the Wharton's jelly of the human umbilical cord. Stem Cells 22 $1330-1337,2004$

19. Fisher LW, McBride OW, Termine JD, et al: Human bone sialoprotein. Deduced protein sequence and chromosomal localization. J Biol Chem 265: 2347-2351, 1990.

20. Avila RE, Carmo RA, Farah Kde P, et al: Hyaluronic acid in the evaluation of liver fibrosis in patients with hepatitis $\mathrm{C}$ on haemodialysis. Braz J Infect Dis 14: 335-341, 2010.
21. Xu XM, Yuan GJ, Deng JJ, et al: Hepatic oval cells activated by hepatocyte apoptosis in diethylnitrosamine-induced rat liver cirrhosis. Saudi Med J 31: 490-494, 2010

22. Barry FP and Murphy JM: Mesenchymal stem cells: clinical applications and biological characterization. Int J Biochem Cell Biol 36: 568-584, 2004

23. Parekkadan B and Milwid JM: Mesenchymal stem cells as therapeutics. Annu Rev Biomed Eng 12: 87-117, 2010.

24. Thapa BR and Walia A: Liver function tests and their interpretation. Indian J Pediatr 74: 663-671, 2007.

25. Dai LJ, Li HY, Guan LX, et al: The therapeutic potential of bone marrow-derived mesenchymal stem cells on hepatic cirrhosis. Stem Cell Res 2: 16-25, 2009.

26. Chamberlain J, Yamagami T, Colletti E, et al: Efficient generation of human hepatocytes by the intrahepatic delivery of clonal human mesenchymal stem cells in fetal sheep. Hepatology 46: 1935-1945, 2007.

27. Parekkadan B, van Poll D, Suganuma K, et al: Mesenchymal stem cell-derived molecules reverse fulminant hepatic failure. PLoS One 2: e941, 2007.

28. van Poll D, Parekkadan B, Cho $\mathrm{CH}$, et al: Mesenchymal stem cell-derived molecules directly modulate hepatocellular death and regeneration in vitro and in vivo. Hepatology 47: 1634-1643, 2008.

29. Chen X, Li Y, Wang L, Katakowski M, et al: Ischemic rat brain extracts induce human marrow stromal cell growth factor production. Neuropathology 22: 275-279, 2002. 